\title{
CO-OPERATIVE SPRING
}

Compiled by Stuart and Mary Houston, Yorkton.

\begin{tabular}{|c|c|c|c|c|c|c|c|c|}
\hline & 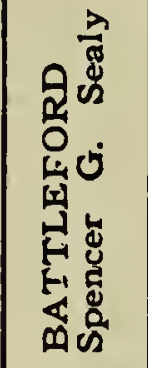 & 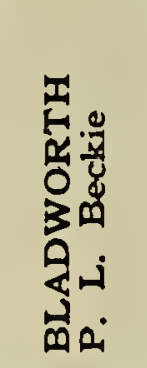 & 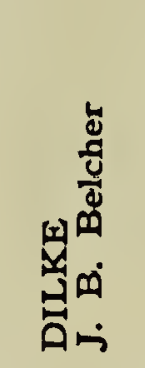 & 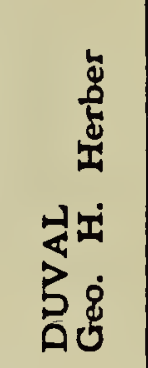 & 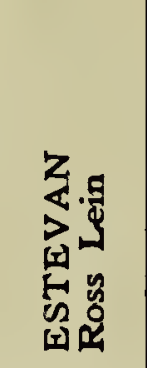 & 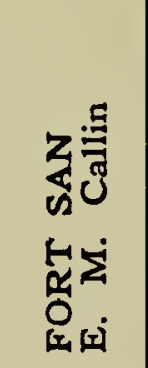 & 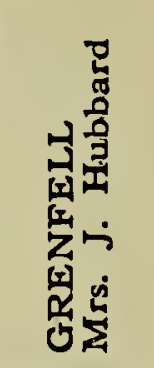 & 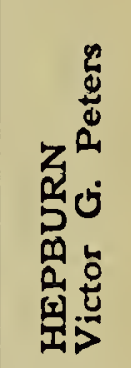 \\
\hline Whistling Swan ..... & Ap18 & Ap18 & Ap16 & Ap2 7 & & Ap16 & Ap30 & \\
\hline Canada Goose ............... & & Ap11 & Mr22 & Mr31 & Ap10 & Mr29 & Ap 6 & \\
\hline 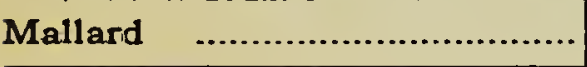 & Mr31 & Mr27 & Mr27 & Mr28 & Mr25 & Mr28 & Mr29 & Mr29 \\
\hline 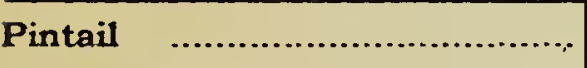 & Ap11 & Mr27 & Mr23 & Mr29 & Mr29 & Ap 9 & Ap 4 & Mr28 \\
\hline 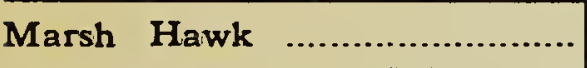 & Mr21 & Mr26 & $\operatorname{Mr22}$ & Mr19 & Ap11 & Mr28 & Mr19 & Mr28 \\
\hline Killdeer $\quad$,................................ & Apr 7 & Ap 1 & Ap 1 & Ap 8 & Mr31 & Mr3 1 & Ap 3 & Ap 9 \\
\hline Common Snipe & & & & Ap26 & & Ap23 & & \\
\hline Mourning Dove $\quad . . \ldots \ldots \ldots \ldots \ldots \ldots . . . . . . . .$. & Ap25 & Ap27 & Ap19 & Ap24 & Ap1 1 & Ap19 & My 2 & My18 \\
\hline Common Nighthawk ................. & My2 1 & My24 & & & My31 & My21 & & My25 \\
\hline Ruby-thraated Hummingbird ... & & My27 & & & & My24 & My25 & \\
\hline Yellow-shafted Flicker ............ & Ap25 & Ap23 & Ap19 & Ap19 & Ap 16 & Ap19 & Ap24 & \\
\hline Eastern Kingbird ... & My 16 & My 17 & My20 & My 8 & My 16 & My 9 & My 14 & My20 \\
\hline Eastern Phoebe ....... & & & & & My 15 & Ap26 & & \\
\hline Barn Swallow ............................. & My 3 & My 9 & My 5 & My 1 & My 4 & My 3 & My 6 & My 13 \\
\hline Purple Martin ......................... & My 7 & & & My20 & & My 3 & My 12 & \\
\hline Common Crow ............................. & Mr21 & Mr21 & Mr21 & Mr20 & Mr21 & Mr21 & Mr22 & Mr22 \\
\hline House Wren ................... & Jn 2 & My29 & Jn 2 & My29 & My 2 & My 6 & My13 & My24 \\
\hline Catbird & $\mathrm{Jn} 4$ & My29 & & My 19 & My25 & My21 & My22 & Jn 3 \\
\hline Brown Thrasher ......................... & My21 & My 19 & My 1 & My 19 & My 16 & My14 & My 18 & My24 \\
\hline Red-eyed Vireo ......................... & My 19 & & & & & My24 & & \\
\hline Yellow Warbler ....................... & My 18 & My 17 & My 15 & My24 & My 3 & My 13 & My 10 & My31 \\
\hline Myrtle Warbler ........... & My 5 & Ap25 & My 9 & & My 2 & Ap26 & My 2 & \\
\hline Redwinged Blackbird $\quad . . . \ldots \ldots \ldots$ & & Ap 1 & Mr31 & Ap 9 & Mr29 & Ap 3 & Ap13 & Ap 8 \\
\hline Baltimore Oriole ....................... & My23 & My20 & My 17 & My19 & My18 & My 17 & My 10 & My27 \\
\hline Rose-breasted Grosbeak ........ & & & & & My22 & My 18 & & \\
\hline American Goldfinch $\ldots . . . \ldots \ldots \ldots$ & My15 & My24 & My27 & My25 & My23 & My16 & My21 & My30 \\
\hline Slate-colored Junco ................. & Ap10 & Ap 8 & Mr27 & & Ap 9 & Mr29 & Ap 10 & \\
\hline Chipping Sparrow ........ & Ap10 & & My 8 & Mr28 & My 11 & My 3 & & \\
\hline White-crowned Sparrow ........ & My14 & Ap28 & My 3 & Ap22 & Ap29 & Ap27 & My 7 & \\
\hline White-throated Sparrow ........ & My 16 & My 4 & My 3 & Ap29 & My 3 & My 2 & My 3 & \\
\hline
\end{tabular}




\section{MIGRATION STUDY, 1959}

\begin{tabular}{|c|c|c|c|c|c|c|c|c|}
\hline & 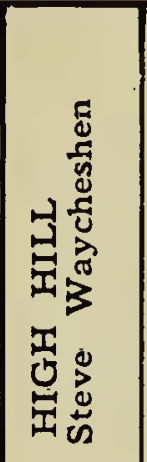 & 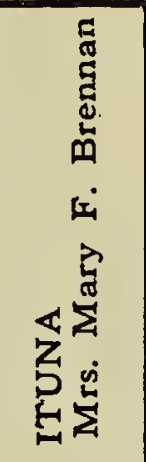 & 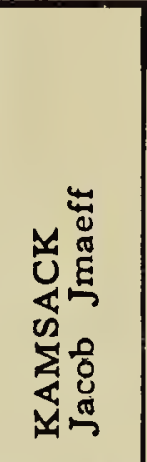 & 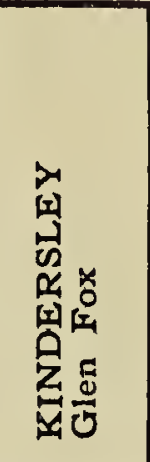 & 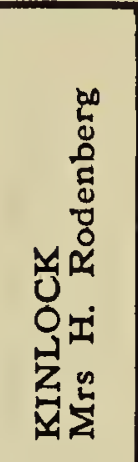 & 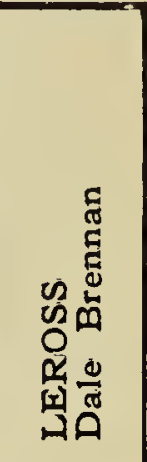 & 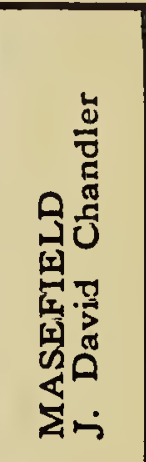 & 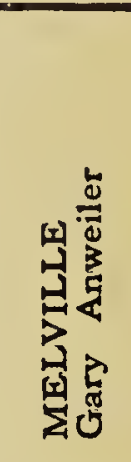 \\
\hline Whistling Swan & My 5 & & & & & & & \\
\hline Canada Groose & Mr28 & & Ap 6 & Mr26 & Ap 2 & Mr31 & Mr28 & \\
\hline Mallard & Mr27 & & Mr29 & Mr27 & Ap 3 & Mr25 & Mr26 & Ap 9 \\
\hline Pintail & Ap13 & & Mr29 & Mr27 & Ap 4 & & Mr25 & Ap 9 \\
\hline Marsh Hawk & Mr24 & & Ap12 & Mr31 & & $\operatorname{Mr} 28$ & Ap 7 & Mr26 \\
\hline Killdeer & Ap11 & Ap 6 & Ap 6 & Mr25 & & Ap 6 & Mr23 & Ap 9 \\
\hline Common Snipe . & Ap27 & & & My 3 & & & & Ap26 \\
\hline Mourning Dove & My 1 & & Ap30 & My 9 & & & Ap26 & My 2 \\
\hline Common Nighthawk & My28 & & & Jn 5 & & & Jn 1 & My 22 \\
\hline Ruby-throated Hummingbird & My27 & $\mathrm{Jn} 12$ & My27 & & & & & My28 \\
\hline Yellow-shafted Flicker & Ap26 & & Ap23 & Ap2 1 & & Ap19 & Ap19 & Ap2 1 \\
\hline Eastern Kingbird & $\mathrm{My} 28$ & My25 & My21 & My16 & My 1 & My19 & My 23 & My 10 \\
\hline Eastern Phoebe $\ldots \ldots \ldots \ldots \ldots \ldots$ & Ap22 & My13 & My 1 & & & Ap 9 & Ap22 & My 19 \\
\hline Barn Swallow …........................ & My 2 & My13 & & My 10 & & My 2 & My 15 & My 9 \\
\hline Purple Martin .......................... & Ap28 & Jn 8 & & & Ap2 8 & & & My 7 \\
\hline 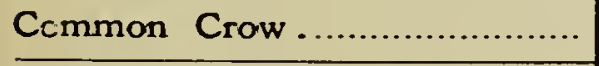 & Mr24. & & Mr23 & Mr23 & Mr24 & Mr19 & Mr16 & Mr26 \\
\hline House Wren - .............................. & My 10 & & My17 & My20 & & My 17 & My11 & My 18 \\
\hline Catbird & Jn29 & My27 & My20 & & & & Jn 7 & My22 \\
\hline 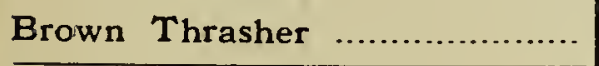 & & My22 & & My20 & & & My23 & My22 \\
\hline Red-eyed Vireo & My24 & & & My26 & & & My 4 & \\
\hline Yellow Warbler …...................... & My 7 & My11 & My17. & My 19 & My 8 & & My17 & My 3 \\
\hline Myrtle Warbler ......................... & Ap27 & & My 5 & Ap30 & My 8 & Ap 8 & & Ap30 \\
\hline Redwinged Blackbird & Ap10 & & Ap11 & Ap 9 & Ap 1 & Ap 4 & Mr31 & Ap 9 \\
\hline Baltimore Oriole & Jn 4 & My25 & & My20 & My 7 & & & My 18 \\
\hline Rose-breasted Grosbeak & My19 & My26 & & Ap23 & & & & My10 \\
\hline American Goldfinch & My27 & My21 & My24 & My27 & My 10 & & My31 & $\mathrm{My} 20$ \\
\hline Slate-colored Junco & Mr30 & & Ap 7 & Ap16 & Ap 7 & Ap 7 & Ap 8 & Mr23 \\
\hline Chipping Sparrow & & My11 & Ap12 & Ap25 & Ap21 & & My 2 & My 3 \\
\hline White-crowned Sparrow & & My 4 & My 2 & My 3 & Ap24 & My 9 & My 2 & \\
\hline White-throated Sparrow & My 7 & & My 5 & My 13 & Ap24 & & & My 2 \\
\hline
\end{tabular}




\section{CO-OPERATIVE SPRING}

Compiled by Stuart and Mary Houston, Yorkton.

\begin{tabular}{|c|c|c|c|c|c|c|c|c|}
\hline , & 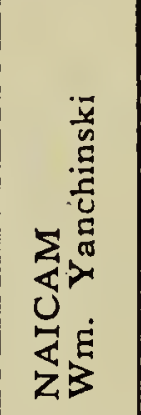 & 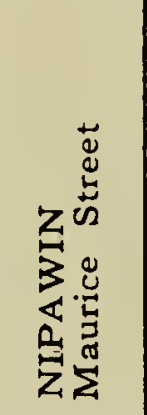 & 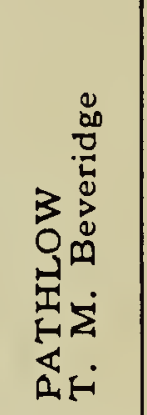 & 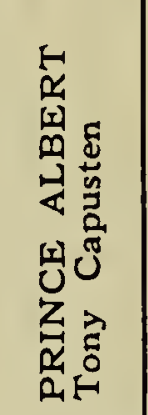 & 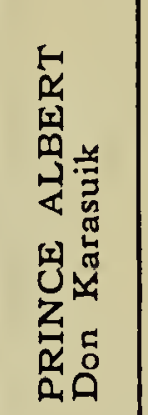 & 要 & 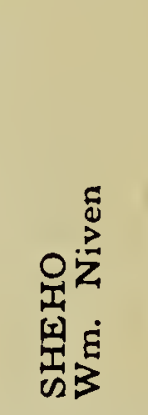 & 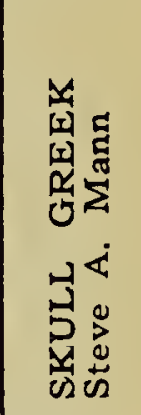 \\
\hline Whistling Swan & & Ap20 & My 3 & & & Mr31 & Ap2 1 & Mr31 \\
\hline Canada Goose & Ap 5 & Ap20 & Ap 3 & Ap 2 & & Mr22 & Mr24 & Mr18 \\
\hline Mallard & Mr29 & Ap 5 & Ap 2 & Ap1 8 & & $\operatorname{Mr} 21$ & Mr27 & Mr24 \\
\hline 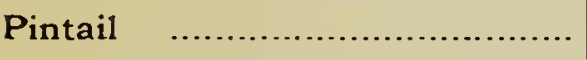 & Ap 5 & Ap 5 & Ap 2 & Ap18 & Mr28 & Mr22 & Mr31 & $\operatorname{Mr} 24$ \\
\hline Marsh Hawk & Mr26 & $\operatorname{Mr} 26$ & Mr26 & & & & Mr25 & Mr27 \\
\hline Killdeer & Mr27 & A.p27 & Ap16 & Ap16 & & Mr26 & Ap 3 & Mr18 \\
\hline Common Snipe .............. & Ap26 & My 8 & Ap2 1 & & My 14 & Ap18 & & Ap19 \\
\hline Mourning Dove & My 9 & My 8 & My 5 & My31 & & Ap18 & My 1 . & Ap26 \\
\hline Common Nighthawk .... & My3 1 & My31 & Jn 2 & $\mathrm{Jn} 3$ & My26 & My27 & My22 & Jn 9 \\
\hline Ruby-throated Hummingbird .. & $\mathrm{Jn} 7$ & My31 & My26 & Jn 2 & My31 & My20 & My30 & \\
\hline Yellow-shafted Flicker & Ap13 & Ap27 & Ap24 & & My 1 & Ap1 1 & Ap24 & Ap10 \\
\hline Eastern Kingbird & My24 & My 16 & & My31 & & My 10 & My 9 & My 16 \\
\hline Eastern Phoebe ...... & My 1 & My 11 & My 2 & My 10 & Ap25 & Ap19 & Ap30 & \\
\hline Barn Swallow …....................... & My 10 & My 9 & & & My 3 & Ap18 & My 6 & My 15 \\
\hline 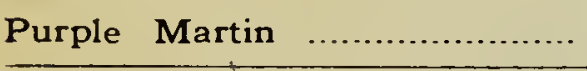 & My 11 & & My 1 & My 5 & My 13 & Ap29 & My 15 & \\
\hline Ccmmon Crow & Mr22 & Mr23 & Mr26 & $\operatorname{Mr} 27$ & Mr23 & Mr 7 & Mr17 & Mr22 \\
\hline House Wren & $\mathrm{Jn} 7$ & My12 & My 11 & My 10 & My22 & My 16 & My 18 & My 16 \\
\hline Catbird & My31 & My29 & Jn 3 & Jn 7 & My29 & My 18 & My 16 & My 5 \\
\hline Brown Thrasher .................. & & & & & & My 15 & My 14 & Jn 4 \\
\hline 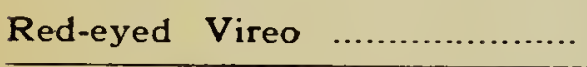 & & My30 & Jn 3 & Jn 7 & Jn 5 & My 16 & My22 & Jn 6 \\
\hline Yellow Warbler ......... & My20 & My25 & My 13 & Ap24 & My 13 & My 10 & My 17 & My 19 \\
\hline Myrtle Warbler ........ & My 6 & Ap29 & Ap24 & My 10 & Ap25 & Ap19 & Ap20 & My 2 \\
\hline Redwinged Blackbird & Ap15 & Ap23 & Ap 6 & Ap18 & & Mr28 & Ap 5 & Ap 9 \\
\hline Baltimore Oriole ......... & My25 & My24 & Jn 3 & & & My 11 & My 16 & My 17 \\
\hline Rose-breasted Grosbeak & & & My 22 & Jn 7 & & My 16 & My25 & \\
\hline American Goldfinch ... & My24 & My31 & Jn 3 & & My24 & My 18 & My 22 & My 19 \\
\hline Slate-colored Junco .... & Ap 7 & Mr31 & Ap 1 & Ap16 & Mr27 & Mr21 & Mr26 & Mr18 \\
\hline Chipping Sparrow & & My 17 & My11. & & My 1 & My 2 & My 12 & Ap 3 \\
\hline White-crowned Sparrow & & My 8 & My 3 & & & Ap2 8 & My 3 & My 1 \\
\hline White-throated Sparrow ... & My 5 & My 6 & My 2 & My 9 & My 8 & My 2 & My 3 & \\
\hline
\end{tabular}




\section{MIGRATION STUDY, 1959}

\begin{tabular}{|c|c|c|c|c|c|c|c|c|}
\hline & 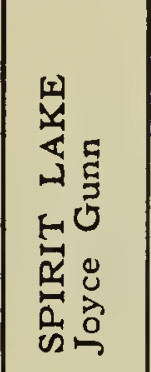 & 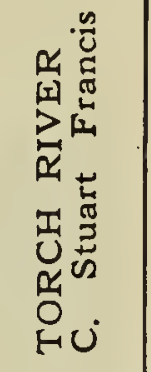 & 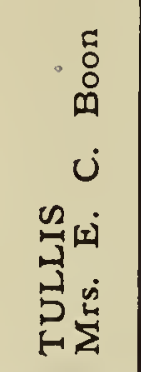 & 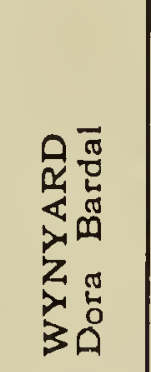 & 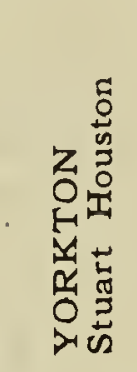 & 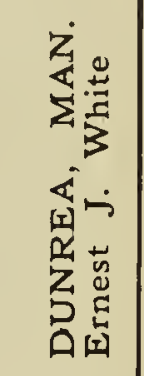 & 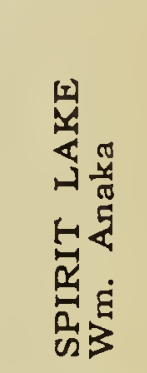 & 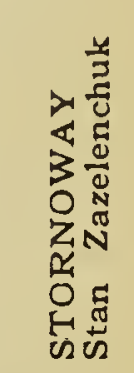 \\
\hline Whistling Swan .... & Ap2 6 & & Ap29 & A p 29 & Ap 7 & Ap1 6 & Ap19 & \\
\hline Canada Goose & Mr26 & My 3 & Mr25 & Mr23 & & $\operatorname{Mr} 27$ & $\operatorname{Mr} 26$ & Ap 3 \\
\hline Mallard $\quad$............... & Ap 2 & Ap 6 & Ap 4 & Ap 3 & Ap 3 & Ap16 & $\operatorname{Mr} 28$ & $\mathrm{Mr} 27$ \\
\hline Pintail $\quad$.................... & Mr31 & Ap13 & Ap 6 & Ap 6 & & & Ap 4 & Ap 3 \\
\hline Marsh Hawk & $\operatorname{Mr} 23$ & Mr31 & $\operatorname{Mr} 27$ & Mr2 1 & $\operatorname{Mr} 27$ & $\operatorname{Mr} 27$ & Mr25 & Mr27 \\
\hline Killdeer & Mr28 & Ap 10 & Ap 6 & Ap 2 & & Mr30 & Ap 1 & Mr30 \\
\hline Common Snipe .... & Ap2 8 & Ap 27 & & & & & Ap14 & \\
\hline Mourning Dove .......... & My 6 & Ap2 7 & My 15 & & My 17 & A p 30 & Ap19 & \\
\hline Common Nighthawk .............. & My27 & My28 & My24 & & My31 & My20 & My24 & \\
\hline Ruby-throated Hummingbird .. & My 17 & Jn 3 & & My26 & & My30 & $\mathrm{Jn} 6$ & \\
\hline Yellow-shafted Flicker $\ldots \ldots \ldots \ldots$ & Ap23 & Ap17 & & Ap20 & Ap 14 & Ap23 & Ap16 & Mr29 \\
\hline Eastern Kingbird $\ldots \ldots \ldots \ldots \ldots \ldots$ & My 9 & My20 & My20 & My20 & My 17 & My 18 & My 9 & My20 \\
\hline Eastern Phoebe ........................ & Ap19 & Ap29 & & & & & Ap20 & Ap30 \\
\hline Barn Swallow $\ldots \ldots \ldots \ldots \ldots \ldots \ldots$ & My 10 & My 9 & My 16 & My20 & My 17 & My 9 & My 3 & My 2 \\
\hline Purple Martin & Ap2 8 & My 20 & & & Ap29 & & & \\
\hline Ccmmon Crow $\ldots \ldots \ldots \ldots \ldots \ldots \ldots$ & Mr2 1 & Mr 9 & Mr22 & Mr2 1 & $\operatorname{Mr} 21$ & $\operatorname{Mr} 21$ & Mr22 & Mr17 \\
\hline House Wren ............................. & My 7 & My27 & Jn 4 & My 14 & My 17 & My 16 & My 15 & \\
\hline Catbird & My20 & & & My30 & My17 & My24 & My25 & \\
\hline Brown Thrasher ..... & & Jn 1 & & My 17 & My 17 & & My 16 & \\
\hline Red-eyed Vireo ............... & & My20 & & & & & My29 & \\
\hline Yellow Warbler & My 7 & & & My23 & My 17 & & My 10 & My 12 \\
\hline Myrtle Warbler .... & Ap24 & & & & My 17 & Ap25 & Ap24 & My 3 \\
\hline Redwinged Blackbird $\ldots \ldots \ldots \ldots$ & Ap 8 & My 4 & Ap 17 & Ap 15 & Mr27 & Ap 1 & Ap 2 & Ap 5 \\
\hline Baltimore Oriole...$\ldots \ldots \ldots \ldots$ & My 20 & My28 & & My23 & My 10 & My 11 & My 10 & My 15 \\
\hline Rose-breasted Grosbeak & & & & & & My23 & My24 & My 12 \\
\hline American Goldfinch $\ldots \ldots \ldots \ldots \ldots$ & My25 & Jn 6 & & My23 & My27 & & My24 & My2 1 \\
\hline Slate-colored Junco ............ & Mr26 & Apj 6 & Ap 4 & Ap 5 & Ap 7 & $\operatorname{Mr} 25$ & Mr27 & \\
\hline Chipping Sparrow $\ldots \ldots \ldots \ldots \ldots \ldots$ & My 8 & Ap20 & & My 16 & My 7 & Ap 5 & My 10 & \\
\hline White-crowned Sparrow ......... & & My 9 & & My 3 & My 2 & My 1 & My 3 & Ap30 \\
\hline White-throated Sparrow ........ & My 3 & My 7 & & My 3 & My 2 & & Ap30 & \\
\hline
\end{tabular}

